\title{
Novel Mutation with Fructose-1,6-Bisphosphatase Deficiency
}

\author{
Madhusudan Samprathi ${ }^{1}$ (D) M. Sridhar ${ }^{1} \cdot$ Rashmi Adiga $^{1} \cdot$ Prakash Vemgal $^{1}$
}

Received: 2 December 2020 / Accepted: 4 February 2021 / Published online: 12 February 2021

(C) Dr. K C Chaudhuri Foundation 2021

To the Editor: Genetic studies in fructose-1, 6-bisphosphatase deficiency (FBP1D) are scarce [1,2]. We report a novel mutation in a developmentally normal, 2-y-old girl, born of a nonconsanguineous marriage, presenting with fever, vomiting, drowsiness, hepatomegaly, hypoglycemia, severe metabolic acidosis, hyperlactatemia $(10.2 \mathrm{mmol} / \mathrm{L})$, normal ammonia, and ketonuria. Neonatal period was unremarkable. One year back, she had a similar illness requiring dextrose and bicarbonate infusion. Tandem mass spectrometry (TMS) was normal. Clinical exome sequencing revealed a homozygous 3 base pair deletion in exon 3 of the FBP 1 gene (chr9:g.94620363 94620365del; Depth: 156x) resulting in an in-frame deletion of amino acids (p.Glu99del; ENST00000415431.5). The FBP1 gene spans approx. $31 \mathrm{~kb}$ on chromosome 9q22.2-q22.3 and consists of 8 exons [1]. The observed variation was in the fructose-1-6-bisphosphatase Nterminal domain of the FBP1 protein, in the nonrepeated region. The p.Glu99del variant has not been reported in the 1000 genomes or in the genome aggregation database (gnomAD). Common variants in the Indian population are missense mutations - c.841G $>$ A (p.Glu281Lys) in exon 8 and c.472C $>\mathrm{T}$ (p.Arg158Trp) in exon 6 [3]. Intragenic deletions in $F B P 1$ are rare $[2,4]$.

FBP1D presents with episodic lactic acidosis and ketotic hypoglycemia, manifesting as hyperventilation, seizures, or coma. Triggers include fever, fasting, vomiting, infections, and excessive fructose ingestion. Half of children have hypoglycemia in the neonatal period, but mostly normal development [1]. Early, prolonged hypoglycemia may cause intellectual disability. Diagnosis is confirmed by identifying biallelic FBP1 pathogenic variants on molecular genetic testing or deficient FBP1 activity in liver or mononuclear white blood cells, the latter not widely available [1].

Madhusudan Samprathi madhu_1511@yahoo.com

1 Department of Pediatrics, Rainbow Children's Hospital, Bannerghatta Road, Bangalore 560076, India
FBP1D should be suspected in children with episodic hypoglycemia, lactic acidosis, and ketosis. TMS is normal; elevated glycerol-3-phosphate in urine organic acid analysis is suggestive [1]. Molecular diagnosis helps in timely and accurate diagnosis of this rare but treatable disorder. Simple measures (avoiding triggers, timely admission, and dextrose infusion) can ensure a normal life for the child.

\section{Declarations}

Conflict of Interest None.

\section{References}

1. Bijarnia-Mahay S, Bhatia S, Arora V. Fructose-1,6-Bisphosphatase deficiency. 2019 Dec 5. In: Adam MP, Ardinger HH, Pagon RA, et al., editors. GeneReviews ${ }^{\circledR}$ [Internet]. Seattle (WA): University of Washington, Seattle; 1993-2020.

2. Santer R, du Moulin M, Shahinyan T, et al. A summary of molecular genetic findings in fructose-1,6-bisphosphatase deficiency with a focus on a common long-range deletion and the role of MLPA analysis. Orphanet J Rare Dis. 2016;11:44.

3. Bhai P, Bijarnia-Mahay S, Puri RD, et al. Clinical and molecular characterization of Indian patients with fructose-1, 6bisphosphatase deficiency: identification of a frequent variant (E281K). Ann Hum Genet. 2018;82:309-17.

4. Lebigot E, Brassier A, Zater M, et al. Fructose 1,6-bisphosphatase deficiency: clinical, biochemical and genetic features in French patients. J Inherit Metab Dis. 2015;38:881-7.

Publisher's Note Springer Nature remains neutral with regard to jurisdictional claims in published maps and institutional affiliations. 